\title{
EFFECT OF THE GRAVITATIONAL ABERRATION ON THE EARTH GRAVITY FIELD
}

\author{
Janusz B. ZIELIŃSKI ${ }^{1}$, Vladimir V. PASHKEVICH ${ }^{2}$ \\ ${ }^{1}$ Space Research Centre, Polish Academy of Sciences, Warsaw, Poland \\ ${ }^{2}$ Central Astronomical Observatory at Pulkovo, Russian Academy of Sciences, \\ St. Petersburg, Russia \\ e-mails:.jbz@,cbk.waw.pl,pashvladvit@yandex.ru
}

\begin{abstract}
Discussing the problem of the external gravitational potential of the rotating Earth, we have to consider the fundamental postulate of the finite speed of the propagation of gravitation. This can be done using the expressions for the gravitational aberration compared to the Liénard-Wiechert solution of the retarded potentials. The term gravitational counteraberration or co-aberration is introduced to describe the pattern of the propagation of the gravitational signal emitted by the rotating Earth. It is proved that in the first approximation, the classic theory of the aberration of light can be applied to calculate this effect. Some effects of the gravitational aberration on the external gravity field of the rotating Earth may influence the orbit determination of the Earth artificial satellites.
\end{abstract}

Keywords: propagation of gravitation, speed of gravity, aberration of gravity, retarded potential, Earth gravitational potential

\section{INTRODUCTION}

In the excellent book Relativistic Celestial Mechanics of the Solar System (Kopeikin et al., 2011), we find the notion aberration of gravity, understood as the effect of the LiénardWiechert retarded potential of the moving mass on the propagation of gravity. It is a very interesting concept, which could be discussed more closely in the context of the classic definitions of the aberration of light and the relativistic gravitational potential. In particular, it might be useful to consider this notion for the case of a rotating body with non-homogeneous mass distribution like the Earth. Some effects of the finite speed of the gravitational propagation on the external gravity field of the rotating Earth have been discussed in Zieliński et al. (2007).

\section{ABERRATION OF LIGHT}

The discovery of the aberration of light is attributed to James Bradley, who presented in 1729 his observations of changes in the positions of stars to the Royal Society. In his analysis, he found that the variation in the apparent position of stars depends on the ratio of the Earth's orbital velocity to the velocity of light. The last one has been determined by Ole C. Rømer a half century earlier from the observation of the eclipses of the satellites of Jupiter. Bradley's results constituted an important contribution to the physics of light and to astronomy. As the Earth's orbital speed is about $30 \mathrm{~km} / \mathrm{s}$, the ratio is of the order of $10^{-4}$. This is referred to as an annual aberration; however, a similar effect will appear for other kinds of motion.

(C) The Author(s). This is an open-access article distributed under the terms of the Creative Commons Attribution License (CC BY 4.0, https://creativecommons.org/licenses/by/4.0/), which permits use, distribution, and reproduction in any medium, provided that the 
The classic Newtonian explanation of the aberration of light could be shown using the plane defined by vectors $\boldsymbol{V}$ and $\boldsymbol{l}$ representing the motion of the observer and motion of the photon from the light source to the observer, respectively. It is tacitly assumed that these vectors are defined in the rest-frame - the primary inertial frame in the sense of the Newtonian absolute space. In optics, the direction of the incoming light ray is determined by the direction of the axis of the telescope - from the center of the object glass to the eyepiece. Because the observer is in motion, it will move by some small line segment, while the photon moves through the telescope. Therefore, the observer will measure the apparent direction ES' instead of the true one ES' (see Figure 1). By simple geometric transform, we can prove that the difference:

$$
\Delta \theta=\theta-\theta^{\prime}=\kappa \sin \theta
$$

in which:

$$
\kappa=(v / c) \operatorname{cosec} 1^{\prime \prime}
$$

$v \quad-\quad$ speed of the observer,

$c \quad-\quad$ speed of light,

$\theta \quad-\quad$ angle between the true direction to the light source and the direction of motion of the observer, and

$\theta^{\prime} \quad-\quad$ apparent direction to the light source.

This is the law of aberration according to the classic approach (W.M. Smart, 1960). The dimension of (1) is second of arc.

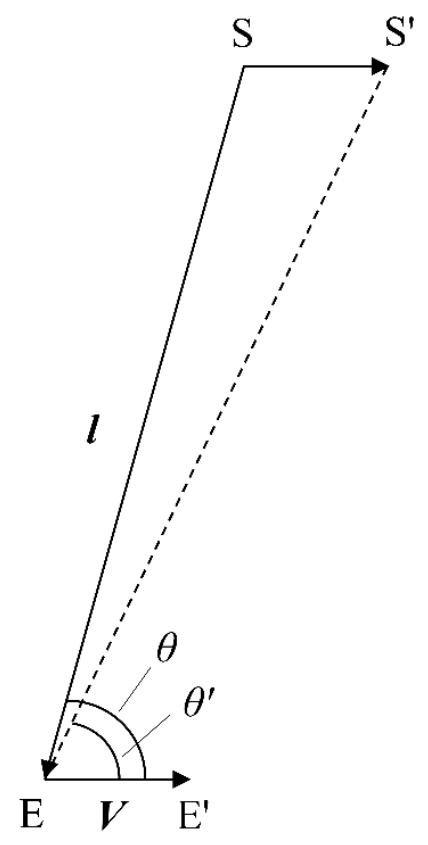

Figure 1. Aberration of light

This law is applied to the orbital motion of the Earth around the Sun but also to the diurnal rotation of the Earth about its axis. In this last case, the speed of the observer depends on the speed of rotation and the latitude of the observer's position:

$$
v_{\varphi}=\frac{2 \pi a \cos \varphi}{86164}\left(\frac{\mathrm{km}}{\mathrm{s}}\right)
$$

where:

a $\quad-$ mean Earth radius in $\mathrm{km}$,

$\varphi \quad-$ latitude of the observer, 
86164 - number of mean solar seconds in a sidereal day.

The coefficient $\kappa_{d}$ for diurnal aberration is:

$$
\kappa_{d}=\left(\frac{0.464}{c}\right) \cos \varphi \operatorname{cosec} 1^{\prime \prime}=0^{\prime \prime} .32 \cos \varphi
$$

The above expressions are derived considering two different reference frames - the inertial frame related to the positions of distant stars and the rotating frame in which the observer with his telescope is located. It is assumed that in the inertial frame, the ray of light (or the photon) is following a straight line, while in the rotating frame, the path of the photon is represented by a curved line.

The use of the inertial frame requires some comment. This notion is well defined in the Newtonian physics; in astrometry, it is defined by directions to distant objects, e.g. quasars. However, in modern geodesy, we are using the local inertial frame connected with the Earth center of mass. We can follow here the definition by Ashby (Ashby, 2004), who writes: "Einstein's Principle of Equivalence allows one to discuss frames of reference which are freely falling in the gravitational fields of external bodies. Sufficiently near the origin of such a freely falling frame, the laws of physics are the same as they are in inertial frame; in particular electromagnetic waves propagate with uniform speed $\mathrm{c}$ in all directions. Such freely falling frames are called locally inertial frames. For the GPS, it is very useful to introduce such a frame that is non-rotating, with its origin fixed at Earth's center, and which falls freely along in the Earth in the gravitational fields of the other solar system bodies. This is called an Earth Centered Inertial (ECI) Frame."

The phenomenon of aberration, originated from the relation of velocities of different reference frames, could be considered in terms of special relativity. In the textbook (Moritz, HofmannWellenhof, 1993), we find an elegant interpretation of the light aberration phenomenon in terms of special relativity. The authors show that only the application of the Lorentz transformation leads to the correct results where the speed of light $c$ is not exceeded. The expression is presented:

$$
\tan \Delta \theta=\frac{v}{c} \frac{1}{\sqrt{1-\frac{v^{2}}{c^{2}}}}
$$

The problem of the aberration of light from the point of view of special relativity is discussed also in the books (Brumberg, 1991; Soffel, 1989). It is discussed in greater detail by Kopeikin (Kopeikin et al., 2011, p. 152) in the frame of the special relativity setting. He got the expression:

$$
\Delta \theta=\frac{v}{c} \sin \theta+\frac{v^{2}}{4 c^{2}} \sin 2 \theta+\frac{v^{3}}{6 c^{3}} \sin \theta\left(1+2 \cos ^{2} \theta\right)+\ldots
$$

The first linear term is the classic formula for aberration of light in terms of the Newtonian approach, same as expression (1). The other terms are special relativistic corrections, which might be important for relativistic geodesy solutions. In the case of the Earth rotation, the ratio $\left(\frac{v}{c}\right)^{2}$ is of the order $10^{-11}$; therefore, the second term should be taken into account if the accuracy of measurements will be comparable.

\section{CO-ABERRATION}

Let us consider the reversed situation. We can imagine that the light signal is emitted from the position of the observer on Earth and is sent into space. Its path in space in the inertial frame is 
described as a straight line, but seen from the Earth in the rotating frame, it will describe a spiral, called the Archimedean spiral (Grøn, 2009, p. 84), (see Figure 2a and 2b).

a.

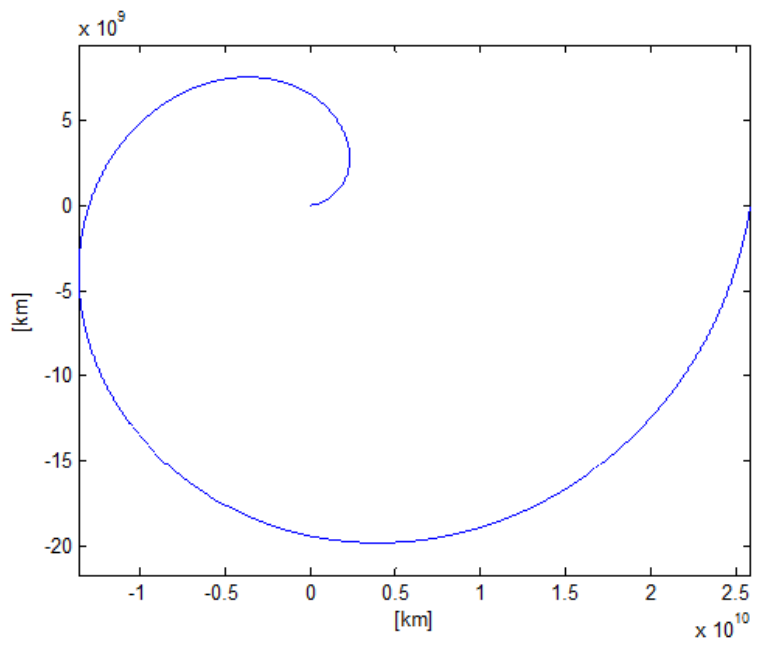

b.

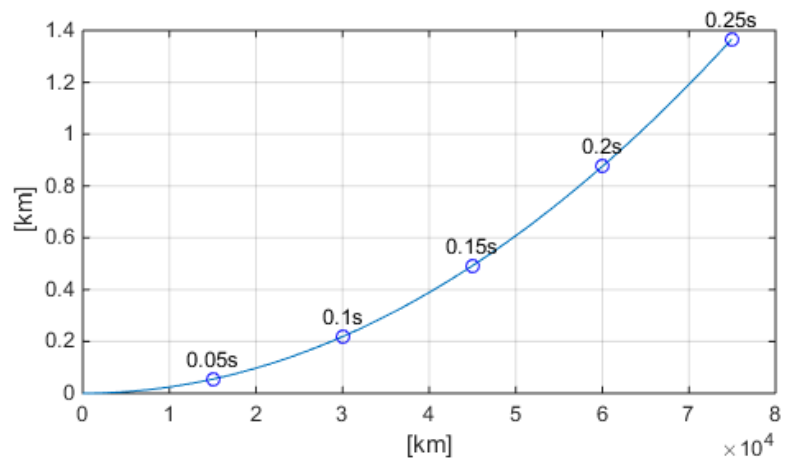

Figure 2. Archimedean spiral

a. photon path in the rotating Earth fixed reference frame for $24 \mathrm{~h}$; b. segment for $0.25 \mathrm{~s}$

Expressions (1)-(3) will be valid, but with the opposite sign. However, these expressions refer to the observer located on the surface of the Earth. If we go into space above the Earth onto altitudes of Earth satellite orbits, the coefficient $\kappa_{d}$ will change. If we imagine a point in space on the altitude of GPS orbits $\left(H_{G P S}=20200 \mathrm{~km}\right)$, but with fixed coordinates $\varphi$ and $\lambda$, we get:

$$
\begin{gathered}
v_{\varphi}=\frac{2 \pi\left(a+H_{G P S}\right) \cos \varphi}{86164}\left(\frac{\mathrm{km}}{\mathrm{s}}\right)=1.938 \cos \varphi \\
\kappa_{d}=\left(\frac{1.938}{\mathrm{c}}\right) \cos \varphi \operatorname{cosec} 1^{\prime \prime}=1^{\prime \prime} .34 \cos \varphi
\end{gathered}
$$

In the inertial frame, the situation is simpler. Let $\alpha$ and $\delta$ be spherical coordinates in the inertial frame of the direction to which is aimed the light ray emitted from the center of the rotating frame. The spherical coordinates of the same direction in the rotating frame are $\varphi$ and $\lambda$. With $T$ the current rotation time and $\omega$ the rotation rate, we have:

$$
\begin{gathered}
\varphi_{T} \equiv \delta, \quad \alpha_{T} \equiv \alpha, \\
\lambda_{T}=\lambda+\omega \cdot \Delta T
\end{gathered}
$$

The distance of the photon racing from the emission point in both frames will be:

$$
r=c \cdot \Delta T
$$

The equations above represent a straight line in the inertial frame but describe the Archimedean spiral in the rotating Earth fixed frame. As we talk about the aberration of the incoming light ray, let us call the analogue process of the outgoing ray the counter aberration or shortly coaberration.

Now, if we assume that the gravity signal - or graviton - follows the same trajectory as the photon, we could talk about the aberration and co-aberration of gravity. Of course, the light and gravitation are not the same, but both can be emitted isotropically and have the same speed. Therefore, similar expressions could be used for both cases. Is this assumption justified?

To answer this question, we have to consider some postulates of special and general relativity. One fundamental principle of this theory is that nothing travels faster than light. This means that changes in the gravitational field cannot be felt everywhere instantaneously: they must 
propagate. According to the relativity theory, they propagate at exactly the same speed as electromagnetic waves in vacuum - the speed of light (Schutz B., F. Ricci, 2001, p.15). Further, photons and gravitational signals leaving the same source at the same time will continue to travel together through the universe, provided they move through vacuum (Schutz B., 2009, p. 212). In addition, this postulate has been confirmed experimentally by the observations of the gravitational waves (LIGO, 2016). So, the answer to the question above is positive - we can compare the propagation of the gravitation with the propagation of the electromagnetic waves.

\section{RETARDED POTENTIAL}

The rotation of the Earth is described with respect to the inertial reference frame. In this frame, we have to use the gravitational field of the rotating Earth. But, the well-known expression used in geodesy to model the Earth gravitational field:

$$
V=\frac{G M}{r}\left[1+\sum_{n=2}^{\infty} \sum_{m=0}^{n}\left(\frac{a}{r}\right)^{n}\left(C_{n m} \cos m \lambda+S_{n m} \sin m \lambda\right) P_{n m}(\sin \varphi)\right]
$$

is valid in the Earth-fixed rotating reference frame, with coordinates $r, \varphi, \lambda$. Here $G$ is the gravitational constant, $M$ is the mass of the Earth, $a$ is the equatorial mean radius of the Earth, $C_{n m}$ and $S_{m n}$ are the numerical coefficients of the model, $P_{n m}$ are the Legendre polynomials of degree $n$ and order $m$.

Such an approximation is justified for low orbits where we can neglect the effect of the propagation of the gravitation. However, for higher orbits such as the GNSS, where the distance from the Earth surface is of the order of $20,000 \mathrm{~km}$, this effect should be considered.

The basic equations for the description of the potential field of the moving body is given in Landau, Lifshitz (2009) as the Liénard-Wiechert potential. Let us consider the gravity potential at the external point $P$, exerted by the point mass $m$ being in motion in the inertial frame. Let

$$
\boldsymbol{R}=\boldsymbol{R}(t)
$$

is the vector defining the position of $m$ as a function of time $t$ (see Figure 3).

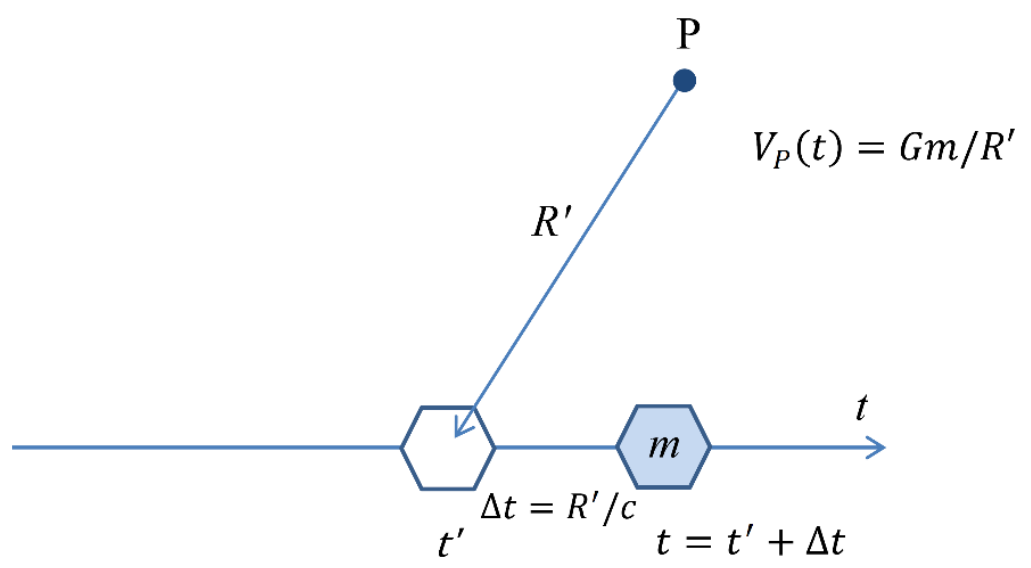

Figure 3. Retarded potential effect

The gravity potential at the point $P(x, y, z)$ is determined by the gravity signal emitted from $m$ in the moment $t^{\prime}$ earlier, such that the time interval necessary to travel by the signal from the point $\boldsymbol{R}\left(t^{\prime}\right)$ to $P$ be equal to $t-t^{\prime}$. Let $\boldsymbol{R}$ be the vector between point $P$ and mass $m$, also function of time and $R=|R|$. Then:

$$
t^{\prime}=t-\frac{R\left(t^{\prime}\right)}{c}
$$


Then, the potential $V_{P}$ generated by the mass $m$ at the moment $t$ will be:

$$
V_{P}(t)=\frac{G m}{R\left(t^{\prime}\right)}
$$

Following Landau, Lifszyc (2009, p. 203, eq. 63.5,a), for the moving mass $m$, we can write:

$$
V_{P}(t)=\frac{G m}{R^{\prime}-v \cdot \frac{R\left(t^{\prime}\right)}{c}}
$$

where $v$ is the vector of the speed of motion of $m$, and $R^{\prime}$ is the distance between $m$ and $P$ at the time $t^{\prime}$. This is called the Liénard-Wiechert potential.

Looking for an exact solution, we could use the method proposed by Brumberg, Kopeikin (1989) or other authors applying the formalism of the general relativity theory. However, for practical tasks addressing the measurable effects in the motion of the Earth artificial satellites, we may use the approximate solution, taking into account only the speed of the gravity propagation.

Taking the gravity field model (10), we can say that the first and second terms of this expression describe the ellipsoid, while all other terms in the series describe anomalies. These anomalies extend in space (with the growing $r$ - the distance from the mass center).

In the Earth Fixed Reference (EFR) frame, the anomalies have constant coordinates $\varphi, \lambda$ but with extension to space, the $r$ is growing. In the Inertial Earth-centered frame, the coordinate $\lambda$ is replaced by $\alpha$. The Earth is rotating with the rotation speed $\omega$ in the current time $T$. If we want to calculate the anomalies outside of the Earth, we have to take into account the property of the propagation of gravity and the rule of the retarded potential (14). If we assume that gravity is propagating analogously to light then for the increment of time $\Delta T$, the anomaly will be propagated along the straight line up in the inertial frame by the distance $\Delta r=c \cdot \Delta T$ while the Earth will move by the angle $\Delta \alpha=\omega \cdot \Delta t$. It means that in the EFR frame, the anomaly propagates not exactly in the radial direction but is inclined by the increment $\Delta \lambda=-\Delta \alpha$ in the direction opposite to the rotation of the Earth or conforming to the motion of the celestial sphere.

It is interesting to notice that in this approximation, the dependence of $\Delta \lambda$ from $\Delta T$ is linear; therefore, after integration over a time interval $\Delta T=T_{i}-T_{0}$, we have:

$$
\lambda(T)=\lambda_{0}+\omega \cdot \Delta T
$$

that is exactly the same as (8). It shows that in this approximation, the effect of gravitational aberration is analogous to the effect of the retarded potential.

\section{EFFECTS ON THE EARTH GRAVITY FIELD MODEL}

This model, currently used in the form (10), is valid for the EFR frame, with polar coordinates $r, \varphi, \lambda$. On the other hand, solutions in satellite geodesy calculation of orbits are usually done in the Earth-centered inertial frame with coordinates $r, \delta, \alpha$. A simple transformation between these frames is:

$$
\alpha+\tau=\theta_{G r}-\lambda
$$

where:

$\tau \quad-$ hour angle;

$\theta_{G r} \quad-\quad$ the Greenwich sidereal time.

When the gravity signal, generated by the motion of the non-homogeneous Earth mass, is emitted in space at the moment $T_{0}$, it follows the vertical straight line in the inertial frame with 
the speed of light $c$. The direction of this straight line is defined by the coordinates $\delta_{0}, \alpha_{0}$. After the increment of time, at the moment $T_{i}$, it will reach the distance:

$$
R_{i}=c \cdot\left(T_{i}-T_{0}\right)=c \cdot \Delta T_{i}
$$

At the same time, the Earth will rotate by the angle:

$$
\Delta \alpha=\omega \cdot \Delta T_{i}
$$

what reflects the gravitational co-aberration, according to (15).

Therefore, the potential field at the distance $r_{i}$ will be retarded by $\Delta \alpha$ with respect to the initial orientation of the Earth and the direction of emission $\delta_{0}, \alpha_{0}$. The expression (10), if assuming finite propagation speed of gravity according to (17) and substituting (18), should have the form:

$$
V=\frac{G M}{r}\left[1+\sum_{n=2}^{\infty} \sum_{m=0}^{n}\left(\frac{a}{r}\right)^{n}\left(C_{n m} \cos m(\lambda-\Delta \alpha)+S_{n m} \sin m(\lambda-\Delta \alpha)\right) P_{n m}(\sin \varphi)\right]
$$

How could this expression be interpreted? The term $\Delta \alpha$ has small value at distances of the Earth satellite orbits. For satellite navigation orbits with altitude ca. $20000 \mathrm{~km}$, the parameter $r$ is approximately equal:

$$
r=a+R
$$

where $R$ is the distance from the mass point located on the surface of the Earth to the point in space. Following (17), we have:

$$
r=a+c \cdot \Delta T
$$

It means that for all terms under the summation sign, we will have in the denominator the value $c$ raised to the second or higher power. It is conforming to the conclusion of Lorentz that the aberration of force acting between two moving particles exists, but it its effect must be of the quadratic order $\frac{v^{2}}{c^{2}}$ (Kopeikin et al., 2011, p. 184).

It should also be noted that only these terms of expression (19) are relevant in the present discussion, which disturb the symmetry of the field with respect to the axis of rotation. It means that the terms with the index $m=0$ (e.g. $C_{20}, C_{40}$, which determine the ellipsoidal flattening of the Earth) have no influence on the effect of co-aberration.

The term $\Delta \alpha$ is small at distances of the Earth satellite orbits. The order of magnitude is $10^{-11}$. Does it mean that it is negligible? The accuracy of the time measurement is of the order of fraction of a nanosecond. The modern technologies like Inter-Satellite Links (Kur et al., 2020) or Cold Atom Interferometry (Travagnin, 2020) open the way to a further increase of the precision of positioning. Our results claim that the external Earth gravity field will be slightly twisted, provoking small perturbations of some orbits. The most sensitive might be orbits with altitudes $20,000 \mathrm{~km}-40,000 \mathrm{~km}$; however, it requires further investigation. If such perturbations could be observed and measured, two results could be reached: 1) the improvement of accuracy in satellite geodesy solutions and 2) independent experimental corroboration of the value of the speed of the gravitational interaction.

\section{ADDITIONAL REMARKS}

The problem of the gravitational aberration is discussed in deep by Kopeikin and Fomalont in context of the works devoted to the determination of the speed of the gravitational propagation (Fomalont, Kopeikin, 2003; Kopeikin, Fomalont, 2006; Kopeikin, Fomalont, 2007). This famous experiment allowed for the first time in the history of physics to measure with high 
reliability the speed of gravity. The authors used the opportunity of the transit of Jupiter in front of quasar emitting radio waves. VLBI observations of the radio signals enabled to determine the change in quasar position, as the gravitational field of Jupiter bent the incoming radio waves. To analyse the data and to compare the results with the theory, a number of effects had to be identified, among them, the gravitational aberration effect. In the last of the above papers, we find the sentence: The direction of the gravity force propagation changes (aberrates) when one goes from one frame to another. The magnitude of the aberration of gravity effects is linear with respect to $\frac{v}{c_{g}}$ like the aberration of light is linear with respect to ratio $\frac{v}{c}$. The authors used the mathematical approach known as the theory of the Liénard-Wiechert potential of the moving body, in perfect agreement with the Lorenz transformation of the gravity field variables.

In the last years, the gravitational waves research provided the further confirmation of the identity of the speed of light and the speed of the gravitational waves. The gravity observatories LIGO and Virgo observed the gravity event in space - the merger of the pair of the binary black holes, at the distance of above 1 billion of light years from the Earth. The gravity signal and the light signal were observed on the Earth with the difference less than $1 \mathrm{~s}$ (LIGO, 2016).

\section{CONCLUSIONS}

It is shown that we can understand the notion of co-aberration of gravity as an effect produced by the diurnal rotation of the Earth and the finite speed of the propagation of gravitation. However, the above discussion is limited to aspects interesting from the geodetic point of view. The existence of the gravitational co-aberration generated by the mass of the rotating Earth should be taken into account in the high-accuracy solutions in satellite geodesy.

Acknowledgements. This work has been done in the frame of the Agreement of Cooperation between the Space Research Centre of the Polish Academy of Sciences and the Central (Pulkovo) Astronomical Observatory of the Russian Academy of Science. Assistance in computations by Maciej Kalarus is acknowledged.

\section{REFERENCES}

Ashby N. (2004). The Sagnac effect in the Global Positioning System. in Rizzi, G., Ruggiero, M.L.(eds) Relativity in Rotating Frames, series Fundamental Theories of Physics vol.135, pp.11-28, Kluwer.

Brumberg V.A. (1991). Essential Relativistic Celestial Mechanics. Taylor \& Francis Group.

Brumberg V.A., Kopeikin S.M. (1989). Relativistic reference systems and motion of test bodies in the vicinity of the Earth. Il Nuovo Cimento, 103B, pp.63-98.

Fomalont E.B., Kopeikin S.M. (2003). The measurement of the light deflection from Jupiter: Experimental results. Astrophysical Journal, 598, pp.704-711.

Grøn Ø. (2009). Lecture Notes on the General Theory of Relativity. Lecture Notes in Physics. 772, Springer Science-Business Media.

Kopeikin S., Efroimsky M., Kaplan G. (2011). Relativistic Celestial Mechanics of the Solar System. Wiley VCH.

Kopeikin, S.M., Fomalont, E.B. (2006). Aberration and the Fundamental Speed of Gravity in the Jovian Deflection Experiment. Foundations of Physics, 36, pp.1244-1285.

Kopeikin, S.M., Fomalont, E.B. (2007). Gravimagnetism, causality, and aberration of gravity in the gravitational light-ray deflection experiments. General Relativity and Gravitation, 39, pp.1583-1624. 
Kur, T., Liwosz, T., Kalarus, M. (2020) The application of inter-satellite links connectivity schemes in various satellite navigation systems for orbit and clock corrections determination: simulation study. Acta Geod Geophys (2020). https://doi.org/10.1007/s40328-020-00322-4

Landau L.D., Lifszyc J.M. (2009). Teoria Pola. PWN Warszawa.

Landau, L.D., Lifshitz, E.M. (1975) The classical theory of fields. Oxford Pergamon Press.

LIGO Scientific Collaboration and Virgo Collaboration: Abbott, B. P. et al., (2016). GW151226: Observation of Gravitational Waves from a 22-Solar-Mass Binary Black Hole Coalescence. Physical Review Letters. 116 (24)

Moritz H., Hofmann-Wellenhof B. (1993). Geometry, Relativity, Geodesy. Wichmann.

Schutz B. (2009) A First Course in General Relativity. Cambridge University Press.

Schutz B., Ricci F. (2001) Gravitational Waves, Sources and Detectors. In: Gravitational Waves edited by Ciufolini, Gorini, Moschella, Fre, Institute of Physics Publ., Bristol \& Philadelphia.

Smart W.M. (1960). Text-Book on Spherical Astronomy. Cambridge Univ. Press.

Soffel M.H. (1989). Relativity in Astronomy, Celestial Mechanics and Geodesy. Springer Verlag.

Travagnin M. (2020) Cold atom interferometry for inertial navigation sensors. Publ.Office of EU, Luxembourg.

Zieliński J.B., Gałązka R.R., Peron R. (2007). On Possible Determination of the Speed of the Gravity Signal in Space With Help of Gradiometry. Artificial Satellites, 42, pp.120-140.

Received: 2020-07-28

Reviewed: 2020-10-09 (undisclosed name) and 2020-11-23 (undisclosed name)

Accepted: 2021-03-18 